\title{
Oxyresveratrol protective effects against deoxynivalenol-induced intestinal barrier dysfunction and bacterial translocation on porcine intestinal epithelial IPEC-J2 cells
}

\author{
Murphy L.Y. Wan ${ }^{\mathrm{a}, \mathrm{c}}$, Ka Ho Ling ${ }^{\mathrm{a}, \mathrm{c}}$, Hani El-Nezami ${ }^{\mathrm{a}, \mathrm{b}}$ and Mingfu Wang* \\ aSchool of Biological Sciences, Faculty of Science, Kadoorie Biological Sciences Building, The University of Hong Kong, Pokfulam, \\ Hong Kong \\ b Institute of Public Health and Clinical Nutrition, University of Eastern Finland, Kuopio, Finland \\ cMurphy LY Wan and Ka Ho Ling contributed equally to this work. \\ *Corresponding author: Mingfu Wang, PhD, Associate Professor, School of Biological Sciences, 7N-16, Kadoorie Biological Sciences \\ Building, The University of Hong Kong, Pokfulam Road, Hong Kong. Tel: +852 2299 0338; Fax: +852 2559 9114; \\ E-mail: mfwang@hku.hk \\ DOI: $10.31665 / J F B .2018 .1130$ \\ Received: December 28, 2017; Revised received \& accepted: February 06, 2018 \\ Citation: Wan, M.L.Y., Ling, K.H., El-Nezami, H., and Wang, M. (2018). Oxyresveratrol protective effects against deoxynivalenol-induced \\ intestinal barrier dysfunction and bacterial translocation on porcine intestinal epithelial IPEC-J2 cells. J. Food Bioact. 1: 116-123.
}

\begin{abstract}
Deoxynivalenol (DON) is a major mycotoxin contaminant and is known to impair intestinal barrier function. Previous experiments in our laboratory have proven that polyphenols such as resveratrol (RES) may be effective in enhancing epithelial barrier function. Due to the structural similarity of oxyresveratrol (OXY) with RES, it was hypothesized that OXY could also protect against DON-induced intestinal damage. Accordingly, this study aimed to explore potential protective effects of OXY against DON-induced epithelial barrier dysfunction and bacterial translocation on IPEC-J2 cells, in comparison to resveratrol (RES).The results showed that OXY increased transepithelial electrical resistance (TEER) and reduced FD-4 diffusion, whereas DON reduced TEER and increased FD-4 diffusion in IPEC-J2 cells. On the other hand, OXY reduced FD-4 diffusion in DON-damaged cells but showed no significant difference in terms of TEER. Such protective effects coincided with the significantly reduced $E$. coli translocation in cells co-exposed to DON and OXY. Further mechanistic studies demonstrated that OXY protected against DON-induced barrier dysfunction by enhancing the expression of claudin-4 via mitogen-activated protein kinase(MAPK)-dependent pathways. Apparently, OXY worked through the same way as RES did, with results dovetailed nicely with anticipation. These results imply that OXY may share similar health benefits with RES by enhancing epithelial barrier functions and protecting against DON-induced intestinal damage.
\end{abstract}

Keywords: Bacteria translocation; Barrier function; Deoxynivalenol; Oxyresveratrol; Resveratrol; Tight junction.

\section{Introduction}

Mycotoxins are highly toxic low-molecular-weight secondary metabolites of some fungi. Mycotoxin contamination is a serious worldwide problem, with an estimation of up to $25 \%$ of the world's food crop may be contaminated by mycotoxins (FAO, 2013). Mycotoxins can cause various acute and chronic effects on humans and animals, depending on the species and susceptibility of an animal within a species. In human, the toxicological syndromes caused by ingestion of mycotoxins range from death, induction of cancer, growth impairment and decreased resistance to infection (Zain, 2011).

Deoxynivalenol (DON) is a mycotoxin of the trichothecenes family that is mainly produced by Fusarium graminearum and $F$. culmorum. DON is commonly found in cereals and grains, particularly in wheat, barley, maize, oats and in their products, in 
many temperate regions of Europe, North America and Asia. DON exhibits various toxic effects in humans and animals. Acute high doses of DON exposure elicit abdominal distress, increased salivation, malaise, diarrhea and emesis (vomiting) (Peska et al., 2005). Chronic low doses of DON cause anorexia, reduced weight gain, nutrients malabsorption, neuroendocrine changes and immunologic alterations (Peska et al., 2005).

The intestine provides the first physical barrier to protect against ingested toxins and pathogens and is also the first line of defense against intestinal infection. The gut barrier is formed to a large extent by tight junctions that seal the paracellular space between neighboring cells and limit transport of lipids and proteins between the apical and basolateral plasma membranes (Harhaj et al., 2004). Following ingestion of mycotoxin-contaminated food, intestinal epithelial cells (IECs) could be exposed to high concentrations of toxin. Recent reviews by Pinton et al. (2014) and Maresca (2013) have summarized DON induced perturbation of the gut functions in animals and humans(Pinton et al., 2014). Previous studies have also shown that DON can alter intestinal barrier functions in vitro (intestinal epithelial cell line), in vivo (animals) and ex vivo (intestinal explants) (Diesing et al., 2012; Pinton et al., 2009; Pinton et al., 2012). A mechanistic study using IPEC-1 cells demonstrated that DON $(30 \mu \mathrm{M})$ significantly underminedphysical barrier (a drop in TEER) by reducing expression of claudin-4 protein via activation of the p44/42 extracellular signal-regulated kinase (ERK) signaling pathway (Pinton et al., 2010). Such impairment of intestinal integrity could result in subsequent translocation of luminal antigens and bacteria, which could have major implications for human health in terms of sepsis and inflammation (Pinton et al., 2014).

Polyphenols are widespread constituents of fruits, vegetables, cereals, dry legumes, chocolate, and beverages, such as tea, coffee, or wine. Many polyphenols can impact the biological system and can prevent diseases associated with oxidative stress, such as cardiovascular diseases, cancers, inflammation, neurodegenerative diseases and diabetes (Scalbert et al., 2005). For example, resveratrol $\left(3,4^{\prime}, 5\right.$-trihydroxy-trans-stilbene) (RES) is a stilbenoid present abundantly in red wine and grape skin (Walle et al., 2004) and has been extensively studied. It has been known to provide multiple desirable bioactivities such as antioxidative, anti-inflammatory, cancer chemopreventive, and anti-aging (Pirola et al., 2008) and may be useful in treating cardiovascular diseases, cancers, pain, inflammation, tissue injury, and in reducing the risk of neurodegenerative disorders ( $\mathrm{Li}$ et al., 2012). Studies of RES-mediated effect on intestinal barrier function remain very limited, and only few studies have shown that RES ameliorates small intestinal and colonic inflammation in mice by reducing different pro-inflammatory cytokine expression and secretion (Bereswill et al., 2010; SanchezFidalgo et al., 2010). RES has a number of naturally occurring analogs (Figure 1), which are produced by diverse plant species, such as grape, mulberry and peanut. These derivatives differ mainly in the number and position of hydroxyl groups, leading to variation in solubility, stability, bioavailability and antioxidant activity ( $\mathrm{He}$ et al., 2013). For instance, oxyresveratrol (OXY) (trans-2,3',4,5'tetramethoxystilbene) is a hydroxylated analog of RES and is present mainly in Morus alba bark(Shin et al., 1998). OXY is a multi-function bioactive compound: it shows neuroprotective (Chao et al., 2008), depigmenting (skin whitening) (Kim et al., 2002), anti-inflammatory (Chen et al., 2013), as well as anti-viral effects (Galindo et al., 2011). OXY is structurally similar to RES, which has an additional hydroxyl group. Indeed, OXY shows comparable physiological properties to RES in various studies (Li et al., 2015; Xu et al., 2014), and it is suggested to be superior to RES because of its higher water-solubility and lower toxicity (Andrabi et al., 2004). Thus, OXY is a potential substance to be utilized to produce

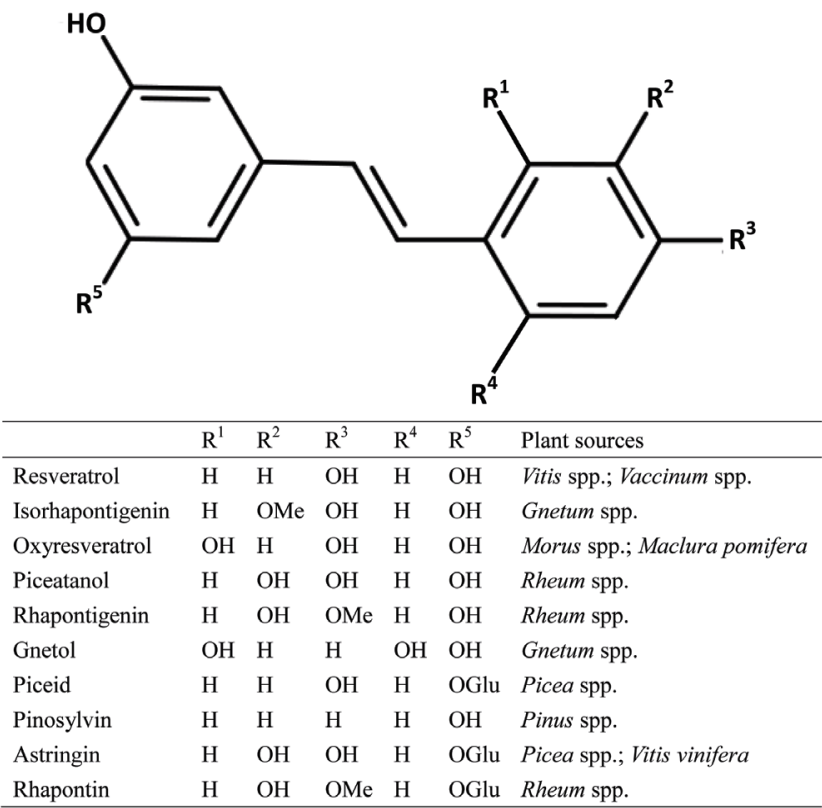

Figure 1. Chemical structures of RES and its natural derivatives/analogues.

medicines, cosmetics and health supplements. Although OXY has been found to possess a wide range of pharmacological properties and of high value in health promotion, studies on OXY-mediated beneficial effects on gut health remains very limited.

Owing to the fact that DON and OXY are food-derived components, they may be present simultaneously in the gastrointestinal tract. There is a potential interactive effect of DON with OXY, which may result in unexpected intestinal effects in intestine. A previous study has demonstrated that co-exposure of DON and RES protected Caco-2 cells from cytotoxicity (Cano-Sancho et al., 2015) and it has also previously been shown in our laboratory that RES may be an effective polyphenol enhancing intestinal barrier function. Due to its structural similarity with RES, it was hypothesized that OXY could also protect against DON-induced intestinal damage and bacterial translocation. Accordingly, the aim of this study was to explore the potential protective effects of OXY in comparison with RES against DON-induced intestinal barrier dysfunction and bacterial translocation of the porcine jejuna epithelial cell line, IPEC-J2. The underlying mechanism(s) was/were also elucidated.

\section{Materials and methods}

\subsection{Chemicals and reagents}

DON $(3 \alpha, 7 \alpha, 15$-trihydroxy-12, 13-epoxytrichothec-9-en-8-one) and RES (3,4',5-trihydroxy-trans-stilbene) were purchased from Sigma Chemical Company (St. Louis, MO, USA). They were dissolved in dimethyl sulfoxide (DMSO) (Merck, Darmstadt, Germany), and stored at $-20{ }^{\circ} \mathrm{C}$ before use. OXY (trans-2,3',4,5'tetramethoxystilbene) was extracted and purified from old twig of Marus alba in our group as described previously (Li et al., 2007). Phosphate buffered saline (PBS), sodium bicarbonate (NaHCO3), diethyl pyrocarbonate (DEPC), ammonium persulfate (APS), N, $\mathrm{N}, \mathrm{N}^{\prime}, \mathrm{N}^{\prime}$-tetramethyl-ethane-1,2-diamine (TEMED), acrylamide, $4 \mathrm{kDa}$ Fluorescein isothiocyanate-dextran (FITC-dextran), bovine 
serum albumin (BSA), protease inhibitor cocktail, phenylmethylsulfonyl fluoride (PMSF), radio-immunoprecipitation assay (RIPA) lysis buffer and Luria-Bertani (LB) agar were purchased from Sigma. Dulbecco's modified Eagle medium (DMEM)-Ham's F-12 (1:1) w/ HEPES (DMEM/F-12), fetal bovine serum (FBS), penicillin/streptomycin, amphotericin B, $0.25 \%$ (wt/vol) trypsin and Tween 20 were provided by Gibco-Life Technology (Eggenstein, Germany). A cell counting kit-8 (CCK-8) colorimetric assay was purchased from Dojindo (Tokyo, Japan). 2X Laemmli sample buffer, Triton X-100 and 2-amino-2-(hydroxymethyl)-1,3-propanediol (Tris) were provided by BioRad (Richmond, CA, USA). The final concentration of DMSO used as solvent in the culture medium was $0.5 \%$.

\subsection{Cell line and culture conditions}

IPEC-J2 is a nontransformed intestinalcell line originally derived from the jejunums of neonatal, unsuckledpiglets(Schierack et al., 2006) and was a kind gift of Per Torp Sangild (Department of HumanNutrition/Clinical Nutrition, Faculty of Life Science, University ofCopenhagen, Copenhagen, Denmark).Briefly, the cells between passages 90 and 100 were maintained in DMEM/F-12, supplemented with $\mathrm{NaHCO}_{3}, 10 \% \mathrm{FBS}$, penicillin $(100 \mathrm{UI} / \mathrm{mL})$, streptomycin $(100 \mu \mathrm{g} / \mathrm{mL})$, and amphotericin B $(0.5 \mu \mathrm{g} / \mathrm{mL})$. The cells, incubated at $37^{\circ} \mathrm{C}$ in humidified $5 \% \mathrm{CO}_{2}$, were maintained with the media changed three times per week; and were split every two weeks using $0.25 \%(\mathrm{w} / \mathrm{v})$ trypsin after washes with PBS. All cells were screened for mycoplasma contamination prior to use (Lonza, Basel, Switzerland).

\subsection{In vitro bacterial translocation assay}

To measure the bacterial translocation across the intestinal epithelial monolayer, a non-pathogenic clinical strain, Escherichia coli ATCC 25922 (kindly provided by W. C. Yam, Department of Microbiology, The University of Hong Kong), was used. The same strain was also used previously by other investigators for bacterial translocation study (Pinto Jr et al., 2007). Indeed, E. coli is identified to be the most prevalent species in bacterial translocation cases in humans (MacFie, 1997; Sedman et al., 1994). A non-pathogenic strain was used to ensure thatanypenetration across the cell monolayer depended mainly on the barrier integritystatus quo, but not due to impairment induced by bacterial endotoxins (Guo et al., 2013). The IPEC-J2 cells were seeded into a permeable Transwell membrane insert (12-mm diameter, $3.0 \mu \mathrm{m}$ pore size, polyester, Corning, New York, NY, USA) and were grown and differentiated as described previously. After 11 days, cells were washed with PBS and incubated with media without serum and antibiotics for $12 \mathrm{~h}$. The cells were then treated with OXY/RES, administrated into the apical side of the Transwell inserts for $12 \mathrm{~h}$. For assay involving DON-induced damage, the procedures were the same except that $4 \mu \mathrm{M}$ of DON was added $1 \mathrm{~h}$ post OXY/RES administration. After $12 \mathrm{~h}$, E. coli ATCC 25922 was inoculated at $10^{5}$ colony forming unit/ well into the apical side of the cell monolayer. The Transwell plate was then incubated, with mild shaking $(50 \mathrm{rpm})$, at $37{ }^{\circ} \mathrm{C}$ in $5 \% \mathrm{CO}_{2}$. After $2 \mathrm{~h}, 100 \mu \mathrm{L}$ media in the Transwell basolateral compartment were collected and plated on LB agar. The agar plates were incubated at $37^{\circ} \mathrm{C}$ for $18 \mathrm{~h}$ to determine the number of $E$. coli present.

\subsection{Measurement of trans-epithelial electrical resistance (TEER)}

The IPEC-J2 cells, at a density of $5 \times 10^{4}$ cells/well, were seeded into a permeable Transwell membrane insert (12-mm diameter, 0.4 $\mu \mathrm{m}$ pore size, polyester, Corning). The cells were grown and differentiated as described previously. After 11 days, the cells were treated in serum-free media for $12 \mathrm{~h}$, then OXY/RES $(25 \mu \mathrm{M})$ was added into the apical Transwell compartment. At various time points, TEER was measured using an ohm-meter (model EVOM, WPI Inc., Sarasota, FL, USA). Resistance values were recorded in $\Omega$ and calculated in $\Omega \cdot \mathrm{cm}^{2}$ by multiplying the area of the membrane filter. Results were expressed as percentage of the control: \% TEER $=\left(\right.$ TEER $\left._{\text {treatment }} / \mathrm{TEER}_{\text {control }}\right) \times 100 \%$. For DON-induced damage, the procedures were the same except DON $(4 \mu \mathrm{M})$ was added $1 \mathrm{~h}$ post RES administration.

\subsection{Paracellular tracer flux assay}

IPEC-J2 were grown and differentiated in $0.4 \mu \mathrm{m}$ pore inserts and treated with RES for various time points as described previously. FITC-dextran $(4 \mathrm{kDa})$ was dissolved in cell culture medium and used at a final concentration of $0.5 \mathrm{mg} / \mathrm{mL}$ in the apical cell compartment. After $24 \mathrm{~h}$ of incubation the amount of fluorescence was measured in the basal compartment with a fluorimeter (Model VICTOR X3, Perkin Elmer, Courtaboeuf, France), with excitation and emission wavelengths of 490 and $520 \mathrm{~nm}$, respectively. Levels of fluorescence were recorded in relative fluorescence units (RFU). Results were expressed as percentage of the control value: \% cumulative flux of $4 \mathrm{kDa}$ FITC (FD-4) $=$ $\left(\mathrm{RFU}_{\text {treatment }} / \mathrm{RFU}_{\text {control }}\right) \times 100 \%$. For DON-induced damage, the procedures were the same except DON was added $1 \mathrm{~h}$ post OXY/ RES administration.

\subsection{Protein extraction, SDS-PAGE and immunoblotting}

The cells, undergoing the same treatment as described for TEER measurement, were washed with PBS and extracted for detergent-insoluble protein fractions as reported previously (Noda et al., 2012). The cells were first treated with $200 \mu \mathrm{L}$ gentle lysis buffer [1\% Triton X-100 and $5 \mathrm{mM}$ EGTA in $50 \mathrm{mM}$ Tris solution at $\mathrm{pH}$ 7.4], supplemented with protease inhibitor cocktail and PMSF, to yield cell lysates. The cell lysates were then centrifuged at $16,000 \mathrm{~g}$ for $10 \mathrm{~min}$ at $4{ }^{\circ} \mathrm{C}$ to sediment a high-density insoluble fraction that is rich in actin-associated proteins. The supernatant was discarded and the pellet was re-suspended and extracted for proteins using RIPA lysis buffer with protease inhibitor cocktail and PMSF added. Protein concentration was determined by the Bradford protein assay (BioRad, Hercules, CA, USA).

Proteins $(10 \mu \mathrm{g})$ were loaded onto $12 \%$ sodium dodecyl sulfate polyacrylamide gel (BioRad), separated by electrophoresis (SDS-PAGE), and then blotted onto a polyvinylidene difluoride (PVDF) membrane (Millipore, Darmstadt, Germany). The membrane was blocked with $5 \%$ BSA in PBS containing $0.05 \%(\mathrm{v} / \mathrm{v})$ Tween 20 (PBST) buffer. Proteins were probed by immunoblotting with diluted primary antibodies $(1: 1000, \mathrm{v} / \mathrm{v})$ for claudin-4 (\#329400, Gibco-Invitrogen, Grand Island, NY, USA), and GAPDH (\# sc-166646, Santa Cruz Biotechnology, Santa Cruz, CA, USA), in combination with horseradish peroxidase (HRP)-conjugated antimouse IgG (\#170-6516, BioRad) secondary antibodies. The blots were developed using Clarity Western ECL blotting kit (BioRad) and chemiluminescence was detected with a digital imaging system (ChemiDoc XRS+ system with image lab software, BioRad). Quantification was performed by ImageJ software (Ver. 1.48, National Institutes of Health, Bethesada, MD, USA) by densitometric 


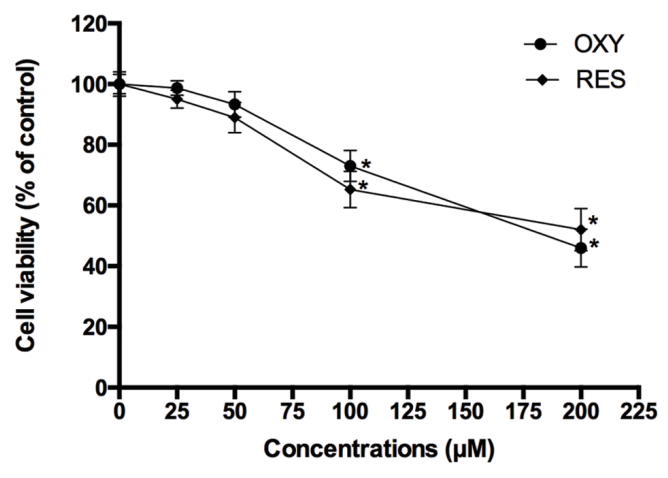

Figure 2. Cytotoxicity of OXY and RES against IPEC-J2 cells. CCK assay was performed on IPEC-J2 cells after $24 \mathrm{~h}$ treatment with increasing concentrations of the stilbenols. Results are presented as percentage of cell viability compared to the control ( $0 \mu \mathrm{M}$ OXY/ RES), values are mean \pm S.D., $n=$ 4. Asterisks indicate a significant difference compared to the control, $P$ $<0.05$.

analysis (Schneider et al., 2012).

\subsection{Kinase phosphorylation assay}

To study the effects of OXY/RES on DON-induced phosphorylation of mitogen-activated protein kinases (MAPKs), quantification of total phosphorylated p38 MAPK, extracellular signal-regulated kinase (ERK), and c-Jun N-terminal kinase (JNK) was performed using a commercial enzyme-linked immunosorbent assay (ELISA) kit (\# ab119674, PhosphoTracer, Abcam) according to the manufacturer's instructions. Cells were seeded in 6-well culture plates (Corning) at $2 \times 10^{5}$ cells/well, and allowed to grow and differentiate for 11 days. After 11 days, the cells were treated with serumfree medium for $12 \mathrm{~h}$. After $12 \mathrm{~h}$, cells were washed and treated firstly with or without $25 \mu \mathrm{M}$ OXY/RES for $1 \mathrm{~h}$, and then with 1 or $4 \mu \mathrm{M}$ DON for $30 \mathrm{~min}$. To determine the optimal time point for the kinase phosphorylation assay, time effects of DON-induced MAPKs phosphorylation were characterized previously (data not shown). Whole cell lysates were collected, normalized for total protein and analyzed for the levels of phosphorylated p38 MAPK, ERK and JNK proteins. Treatments with OXY/RES alone or DMEM were used as controls. All samples were run in triplicate.

\subsection{Statistical analysis}

Data were analyzed using Minitab statistical software (ver. 16, Minitab Inc, State College, PA, USA). Values represent mean \pm standard deviation (S.D.) of at least three independent experiments. Statistical analyses were performed by Student's t-test, Chisquared test, or 1-way ANOVA followed by Tukey's multiple comparison test. $P$ values less than 0.05 were considered significant.

\section{Results}

\subsection{Choice of concentrations of DON, RES and OXY}

The concentrations of DON and RES were chosen based on previous experiments using $4 \mu \mathrm{M}$ DON and $25 \mu \mathrm{M}$ RES as the non-cytotoxic doses (Ling et al., 2016). OXY was also assessed for cytotoxicity

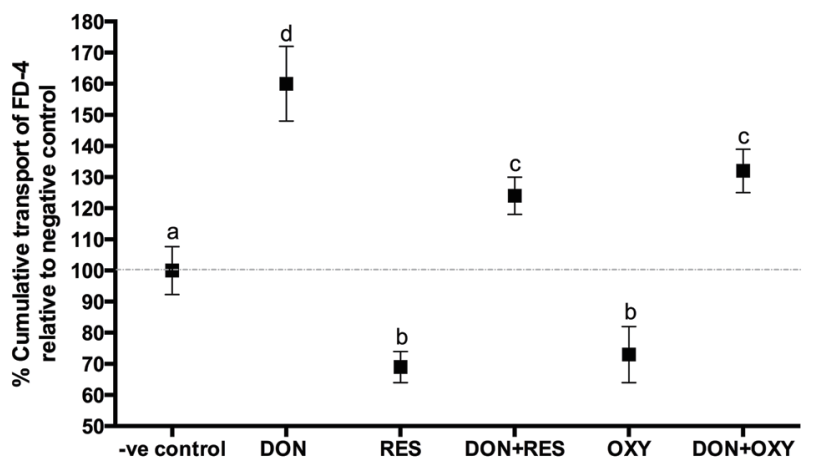

Figure 3. FD-4 diffusion in IPEC-J2 cell monolayers incubated with or without OXY or RES in the presence of absence of DON. Basal FD-4 leve was measured after $12 \mathrm{~h}$. Data are expressed as percentage of FD-4 level relative to the negative control, values are mean \pm S.D., $n=6$. Different letters indicate significant differences $(P<0.05)$ after ANOVA and a post hoc Tukey's multiple comparison test.

using the CCK-8 assay, $50 \mu \mathrm{M}$ was the maximum non-toxic dose (Figure 2).To compare with the effects of RES found previously (Ling et al., 2016), $25 \mu \mathrm{M}$ OXY was used for all subsequent assays.

\subsection{OXY fortified intestinal barrier and protected against DON induced damage}

To determine if OXY protected a cell monolayer from DON-induced intestinal barrier damage, the protective effect of OXY on TEER and paracellular permeability was first assessed and compared with that of RES. A $25 \mu \mathrm{M}$ RES/OXY was administrated to the cells $1 \mathrm{~h}$ prior to challenge with $4 \mu \mathrm{M}$ of DON, TEER and 4-kDa FITC-dextran (FD-4) diffusion were measured after $12 \mathrm{~h}$. The results showed that both RES and OXY increased TEER and reduced FD-4 diffusion, whereas DON reduced TEER and increased FD-4 diffusion in IPEC-J2 cells. On the other hand, both RES and OXY reduced FD-4 diffusion in DON-damaged cells (Figure 3) but showed no significant difference in terms of TEER (Figure 4).

\section{3. $O X Y$ reduced DON-inducedbacterial translocation}

The effect of OXY on DON-induced bacterial translocation was subsequently studied. After 12 h, E. coli was added. Medium (100 $\mu \mathrm{L}$ ) in the basolateral compartment was collected after $2 \mathrm{~h}$, and plated to determine if bacteria were present. The presence of $E$. coli in the basolateral compartment was deemed positive for bacterial translocation. The 2-h incubation time was determined previously in our laboratory, which demonstrated that it took at least $2 \mathrm{~h}$ for the $E$. coli to penetrate about $25 \%$ untreated cell monolayers(data not shown). In the present study, the results showed that similar to RES, OXY significantly reduced (by $36 \%$ ) DON-induced bacterial translocation (Figure 5).

\subsection{OXY enhanced claudin-4 expression and prevented DON-} induced decreased expression of claudin-4

Immunoblot analyses revealed that both OXY and RES enhanced the protein expression of claudin-4 in untreated IPEC-J2 cells (Figures $6 \mathrm{~A}$ and $6 \mathrm{~B}$ ); and reduced DON-induced decreased expression of claudin-4 (Figures 7A and 7B). Apparently, OXY worked through the 


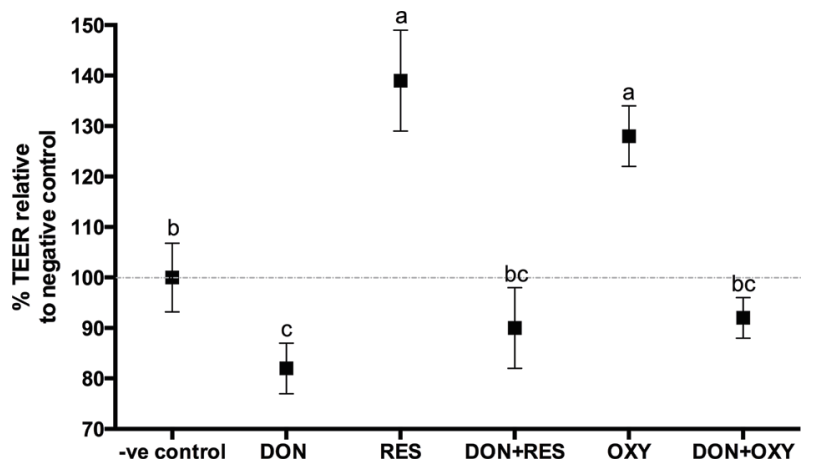

Figure 4. Variation of TEER in IPEC-J2 cell monolayers incubated with or without OXY or RES in the presence or absence of DON. TEER was measured after $12 \mathrm{~h}$. Data are expressed as percentage of TEER relative to the negative control, values are mean \pm S.D., $n=6$. Different letters indicate significant differences $(P<0.05)$ after ANOVA and a post hoc Tukey's multiple comparison test.

same pathway as RES did, with results nicely fit with anticipation.

\section{5. $O X Y$ protected against $D O N$-induced barrier dysfunction via MAPK-dependent mechanisms}

We recently demonstrated that RES protected against DON-induced decreased claudin-4 expression through mitogen-activated protein kinase(MAPK)-dependent pathways. In the present study, we further investigated if OXY also protected against DON-induced decreased claudin-4 expression via MAPK pathways. Cell monolayers were pre-treated with $25 \mu \mathrm{M}$ OXY for $1 \mathrm{~h}$, then cotreated with DON for $30 \mathrm{~min}$. Proteins were then immediately extracted for ELISA analysis. The results showed that OXY significantly reduced DON-activated phosphorylation of $\mathrm{p} 38$ (for $1 \mu \mathrm{M}$

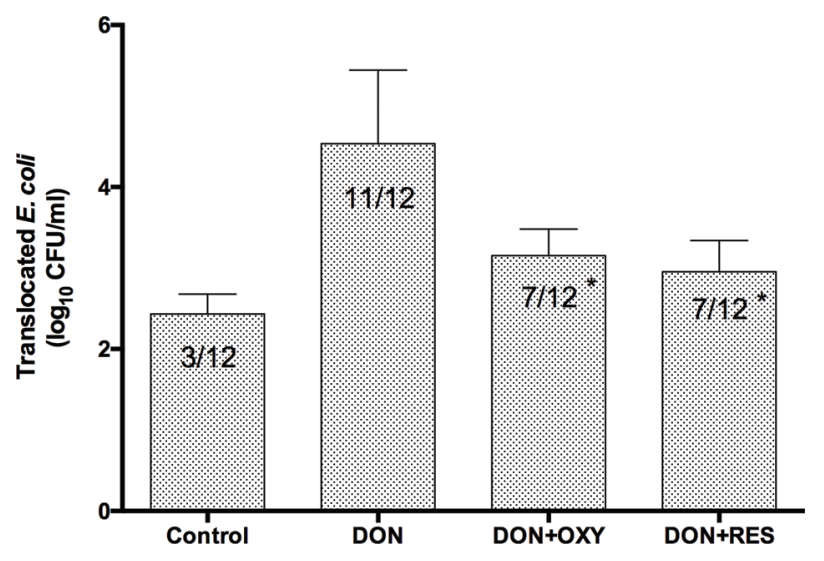

Figure 5. Protective effects of OXY or RES against DON-induced E. coli translocation across IPEC-J2 cell monolayers. $E$. coli were added on the apical side and, after $2 \mathrm{~h}$, media from the basal compartment were plated. Data are expressed as number of $E$. coli determined by plate counting (numbers inside bars indicate number of insert positive for bacterial translocation compared to the total number of epithelial preparations observed). Chi-squared tests were performed to determine the protective effects on bacterial translocation comparing with DON alone treatments, $n=3$. Asterisks indicate a significant difference statistically $(P<0.05)$ and microbiologically (an order of magnitude difference) between treatment with or without OXY or RES in the presence of DON.
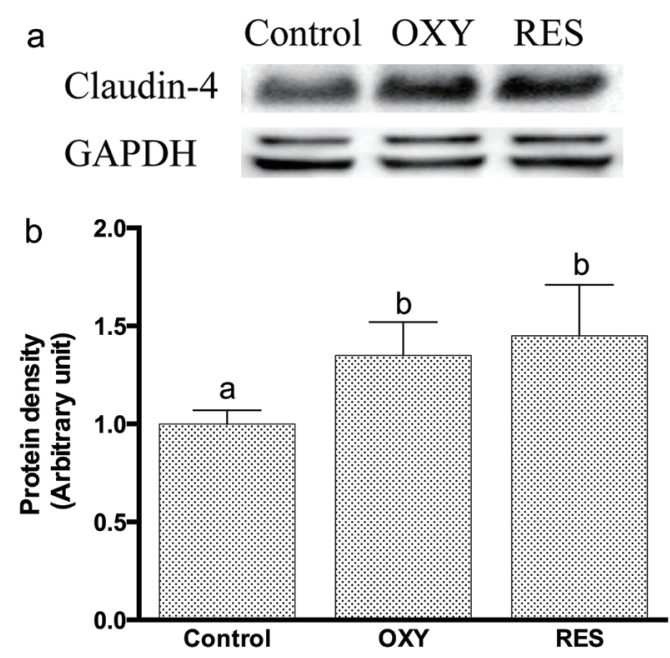

Figure 6. (A) Immunoblot of claudin-4 in the detergent-insoluble fractions incubated with OXY or RES for $\mathbf{1 2} \mathrm{h}$. GAPDH acts as protein loading control; (B) Densitometric analyses of immunoblots of CLN-4 in the detergent-insoluble fractions incubated with OXY or RES for $\mathbf{1 2} \mathbf{~ h}$. The density values were normalized to the negative control after normalization with the loading control (GAPDH). Values are mean \pm S.D., $n=4$. Means without a common letter differ, $\mathrm{P}<0.05$.

DON) and JNK (for both 1 and $4 \mu \mathrm{M}$ DON) (Figures 8A \& 8B), but not ERK (Figure 8C). Again, OXY showed inhibition pattern alike to that of RES found previously in our laboratory (data not yet published), in which p-JNK was the one most reduced.

\section{Discussion}

Polyphenols constitute a large chemical class of phytochemicals among which the stilbenoid sub-class has received growing inter-

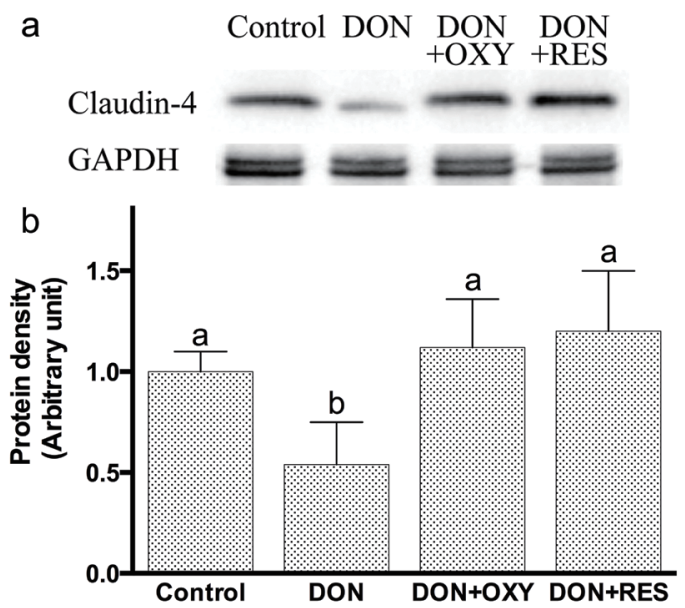

Figure 7. (A) Immunoblot of claudin-4 in the detergent-insoluble fractions incubated with OXY or RES in the presence of DON for $12 \mathrm{~h}$. GAPDH acts as protein loading control; (B) Densitometric analyses of immunoblots of claudin-4 in the detergent-insoluble fractions incubated with OXY or RES in the presence of DON for $\mathbf{1 2} \mathbf{h}$. The density values were normalized to the negative control after normalization with the loading control (GAPDH). Values are mean \pm S.D., $n=4$. Means without a common letter differ, $P<0.05$. 

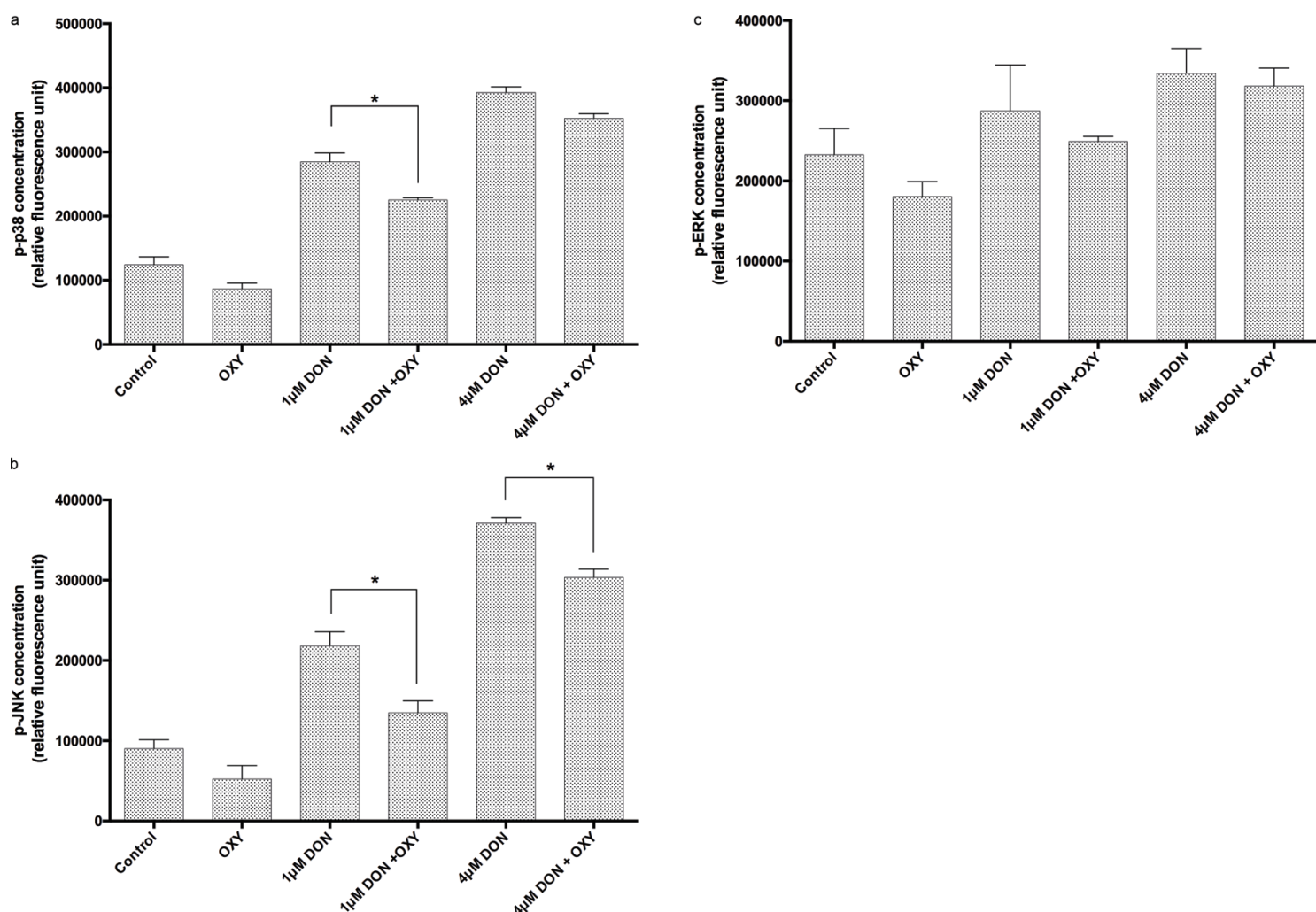

Figure 8. Levels of (A) p38 phosphorylation, (B) JNK phosphorylation and (C) ERK phosphorylation in IPEC-J2 cells treated with or without OXY in the presence of DON. Proteins were extracted $30 \mathrm{~min}$ after DON administration. Data are mean \pm S.D. for $\mathrm{n}=3$ in each group. Asterisk indicates a significant pairwise difference between treatments, $\mathrm{P}<0.05$

est due to its elaborate structural diversity and biological activities. The members of stibenoid family differ from each other mainly in the number and position of hydroxyl groups, leading to variation in solubility, stability, bioavailability and antioxidant activity $(\mathrm{He}$ et al., 2013; Wang et al., 1999). Human tissues are only exposed to dietary polyphenols at very low level via the blood, except for gut cells, which are constantly and directly exposed to a relatively high dose of dietary polyphenols (Kroon et al., 2004). Therefore, it is anticipated that dietary polyphenols can exert an immense effect, locally and systemically, upon the gut.However the mechanisms of their protective effects on intestinal barrier functions are not fully understood. Previous work in our laboratory has found that RES might be effective in enhancing the intestinal barrier function and might be used as a novel mean to counteract the intestinal dysfunction and bacterial translocation following mycotoxin exposure (Ling et al., 2016). The results in the present study demonstrate that OXY, a less investigated stilbene, is a highly interesting candidate for the use of promoting intestinal health, which shows similar bioactivity but higher water solubility than RES. We evaluated its effectiveness in enhancing the intestinal barrier and protecting against intestinal damage induced by DON, one of the most commonly occurring mycotoxins in foods and feeds.

In our model system, the evaluation of possible cytotoxic effects of RES and OXY was performed. It was interesting to note that OXY showed comparable cytotoxic effects as RES, which was contradictory to few previous studies which showed lower cytotoxicity of OXY compared to RES in different model systems (Chao et al., 2008; Galindo et al., 2011). Such discrepancy may be due to differences in the concentrations and the cell types used.
Although not yet fully understood, intestinal dysfunction and the increase in bacterial translocation following DON treatment can exert adverse health effects in human and animalsby increasing the susceptibility to infection as well as inflammation and sepsis (Schneider et al., 2012). In this study, we have revealed that OXY elicited potent protective effects against DON-induced intestinal barrier function and bacterial translocation in the IPEC-J2 cells. OXY displayed a comparable effect on enhancing intestinal physical barrier function as RES, as shown by increasing TEER and reducing FD-4 diffusion, in both untreated and DON-treated cells, by almost the same extent. Similarly, pre-treatment of the cell monolayers with OXY, was also found to reduce DON-induced bacterial translocation. All of these suggest a possible protective role of OXY to mitigate barrier dysfunction caused by DON.

The gut barrier is formed to a large extent by tight junctions (TJ), which are multi-protein complexes that link adjacent epithelial cells near their apical border. The presence of TJ that can seal the paracellular space between neighboring cells accounts for the main physical barrier to bacterial translocation. Previous studies have shown the correlation between decreased barrier function and reduced expression of the tight junction protein, particularly claudin-4 in DON-treated intestinal cells (Pinton et al., 2009; Pinton et al., 2010). Such a decrease in claudin-4 protein expression was found to be associated with an increase in permeability of porcine intestinal epithelial cells to pathogenic E. coli (Pinton et al., 2009). In this sense, claudin-4 apparently is a key protein in the preservation of barrier function by establishing a paracellular seal in the intestinal cells (Pinton et al., 2010).

As previously observed,some polyphenols increased TJ protein 
gene expression in the Caco-2 and T84 cells (Amasheh et al., 2008; Rogoll et al., 2010). Similarly, our laboratory has recently demonstrated that RES fortified intestinal barrier and protected against DON induced damage via modulation of claudin- 4 expression. In the present study, the same protocols were followed through for OXY to characterize its effects on intestinal barrier function and to compare with RES. OXY acted similarly as RES which was shown to enhance the claudin- 4 expression and protected against DONinduced decreased claudin-4 expression. Regulation of TJ structure and function involves the MAPK signaling molecules (Matter et al., 2003). MAPK cascades participate in an array of cellular responses, it was shown that $\mathrm{p} 38$ and $\mathrm{JNK}$ cascades function in stress responses, including inflammation and apoptosis (Schaeffer et al., 1999). The exact cellular responses are cell-type or stimuli specific(Oshima et al., 2008). Previously, DON was shown to activate MAPKs in the Caco-2 and IPEC-1 cells (Pinton et al., 2010; Sergent et al., 2006). It was suggested that DONimpaired intestinal barrier function by decreasing the expression of TJ protein such as claudin-4 through a MAPK-dependent mechanism (Pinton et al., 2009). Polyphenols such as OXY, on the other hand, can modulate a series of signaling cascades, including MAPKs, by directly binding to their ATP binding sites, a property determined by the number and the location of hydroxyl groups on the B ring. OXY can therefore penetrate into the cytoplasm and may have a direct modulation effect on MAPK molecules (Chao et al., 2008). Our results clearly demonstrated that OXY significantly reduced DON-induced phosphorylation for $\mathrm{p} 38$ and JNK. Therefore, it indicated that OXY was a prominent inhibitor to DON-induced MAPK activation, which may protect against DON-induced barrier dysfunction by preventing decreased expression of claudin-4. Nevertheless, further research is needed to disentangle the underlying mechanisms.

Owing to the fact that RES and OXY are relatively non-polar, they are lipophilic enough to permeate the GI tract and absorb readily into the blood (Huang et al., 2008). This suggests that they may exert their effects in the intestine very quickly after oral administration. The solubility of RES is, rather limited in aqueous solutions, which provides difficulties for drug formulation. However, OXY is better water soluble, which may contribute to its ability to exert a protective effect over a wider range of concentrations than RES (Lorenz et al., 2003). In addition, OXY is a natural analogue of RES and can easily be obtained from natural sources. All these properties make OXY a promising candidate for pharmaceutical formulations.

Taken together, due to the structural similarity of OXY and RES, they may share similar health benefits by enhancing epithelial barrier functions and protecting against DON-induced intestinal damage. Chronic exposure of DON could result in intestinal damage, which may have severe health consequences in animals and humans in terms of sepsis and inflammation. Polyphenols, such as RES and OXY, may provide a novel intervention strategy to restrict the consequences of DON ingestion. Based on the results presented, it will be of great interest to compare RES and OXY in an in vivo model for protection against DON-induced intestinal damage. Given that OXY represents only one member out of an enormous pool of RES derivatives, it is anticipated that other members may confer similar, or even more potent, effects to intestinal barrier function and may be used as a novel dietary intervention strategy to reduce DON toxicity in humans/animals.

\section{Acknowledgments}

We thank P. T. Sanglid from the Department of Human Nutrition/ Clinical Nutrition, Faculty of Life Science, University of Copen- hagen, Copenhagen, Denmark, for kindly providing the IPEC-J2 cell line. We also thank W. C. Yam from the Department of Microbiology, University of Hong Kong, for providing the E. coli (ATCC 25922) bacterial strain.

\section{Conflict of interest}

The authors have declared no conflicts of interest.

\section{References}

Amasheh, M., Schlichter, S., Amasheh, S., Mankertz, J., Zeitz, M., Fromm, M., and Schulzke, J.D. (2008). Quercetin enhances epithelial barrier function and increases claudin-4 expression in Caco-2 cells. J. Nutr. 138: 1067-1073.

Andrabi, S.A., Spina, M.G., Lorenz, P., Ebmeyer, U., Wolf, G., and Horn, T.F. (2004). Oxyresveratrol (trans-2,3',4,5'-tetrahydroxystilbene) is neuroprotective and inhibits the apoptotic cell death in transient cerebral ischemia. Brain Res. 1017: 98-107.

Bereswill, S., Muñoz, M., Fischer, A., Plickert, R., Haag, L.-M., Otto, B., Kühl, A.A., Loddenkemper, C., Göbel, U.B., and Heimesaat, M.M. (2010). Anti-inflammatory effects of resveratrol, curcumin and simvastatin in acute small intestinal inflammation. PLoS ONE 5: e15099.

Cano-Sancho, G., González-Arias, C.A., Ramos, A.J., Sanchis, V., and Fernández-Cruz, M.L. (2015). Cytotoxicity of the mycotoxins deoxynivalenol and ochratoxin A on Caco-2 cell line in presence of resveratrol. Toxicol. in Vitro 29: 1639-1646.

Chao, J., Yu, M.S., Ho, Y.S., Wang, M., and Chang, R.C. (2008). Dietary oxyresveratrol prevents parkinsonian mimetic 6-hydroxydopamine neurotoxicity. Free Radic. Biol. Med. 45: 1019-1026.

Chen, Y.C., Tien, Y.J., Chen, C.H., Beltran, F.N., Amor, E.C., Wang, R.J., Wu, D.J., Mettling, C., Lin, Y.L., and Yang, W.C. (2013). Morus alba and active compound oxyresveratrol exert anti-inflammatory activity via inhibition of leukocyte migration involving MEK/ERK signaling. BMC Complement. Altern. Med. 13: 45.

Diesing, A.K., Nossol, C., Ponsuksili, S., Wimmers, K., Kluess, J., Walk, N., Post, A., Rothkotter, H.J., and Kahlert, S. (2012). Gene regulation of intestinal porcine epithelial cells IPEC-J2 is dependent on the site of deoxynivalenol toxicology action. PLoS One 7: e34136.

FAO, 2013. Mycotoxins, [cited 28 Feb 2018]. Available from: http://www. fao.org/food/food-safety-quality/a-z-index/mycotoxins/en/.

Galindo, I., Hernaez, B., Berna, J., Fenoll, J., Cenis, J.L., Escribano, J.M., and Alonso, C. (2011). Comparative inhibitory activity of the stilbenes resveratrol and oxyresveratrol on African swine fever virus replication. Antiviral Res. 91: 57-63.

Guo, S., Al-Sadi, R., Said, H.M., and Ma, T.Y. (2013). Lipopolysaccharide causes an increase in intestinal tight junction permeability in vitro and in vivo by inducing enterocyte membrane expression and localization of TLR-4 and CD14. Am. J. Pathol. 182: 375-387.

Harhaj, N.S., and Antonetti, D.A. (2004). Regulation of tight junctions and loss of barrier function in pathophysiology. Int. J. Biochem. Cell Biol. 36: 1206-1237.

He, S., and Yan, X. (2013). From resveratrol to its derivatives: new sources of natural antioxidant. Curr. Med. Chem. 20: 1005-1017.

Huang, H., Zhang, J., Chen, G., Lu, Z., Wang, X., Sha, N., Shao, B., Li, P., and Guo, D.-A. (2008). High performance liquid chromatographic method for the determination and pharmacokinetic studies of oxyresveratrol and resveratrol in rat plasma after oral administration of Smilax china extract. Biomed. Chromatogr. 22: 421-427.

Kim, Y.M., Yun, J., Lee, C.K., Lee, H., Min, K.R., and Kim, Y. (2002). Oxyresveratrol and hydroxystilbene compounds. Inhibitory effect on tyrosinase and mechanism of action. J. Biol. Chem. 277: 16340-16344.

Kroon, P.A., Clifford, M.N., Crozier, A., Day, A.J., Donovan, J.L., and Manach, C. (2004). How should we assess the effects of exposure to dietary polyphenols in vitro. Am. J. Clin. Nutr. 80: 15-21.

Li, E., Tan, H., and Wang, M. (2015). Oxyresveratrol normalized body weight gain and feed efficiency of mice fed high-fat diet. FASEB J. 29(1 Sup- 
plement): 402-408.

Li, F., Gong, Q., Dong, H., and Shi, J. (2012). Resveratrol, a neuroprotective supplement for Alzheimer's disease. Curr. Pharm. Des. 18: 27-33.

Li, H., Cheng, K.W., Cho, C.H., He, Z., and Wang, M. (2007). Oxyresveratrol as an antibrowning agent for cloudy apple juices and fresh-cut apples. J. Agric. Food Chem. 55: 2604-2610.

Ling, K., Wan, L., El-Nezami, H., and Wang, M. (2016). Protective capacity of resveratrol, a red wine constituent polyphenol against deoxynivalenol-induced intestinal barrier dysfunction and bacterial translocation. Chem. Res. Toxicol 29: 823-833.

Lorenz, P., Roychowdhury, S., Engelmann, M., Wolf, G., and Horn, T.F.W. (2003). Oxyresveratrol and resveratrol are potent antioxidants and free radical scavengers: effect on nitrosative and oxidative stress derived from microglial cells. Nitric Oxide 9: 64-76.

MacFie, J. (1997). Bacterial translocation in surgical patients. Ann. R. Coll. Surg. Engl. 79: 183-189.

Maresca, M. (2013). From the gut to the brain: journey and pathophysiological effects of the food-associated trichothecene mycotoxin deoxynivalenol. Toxins 5: 784-820.

Matter, K., and Balda, M.S. (2003). Signaling to and from tight junctions. Nat. Rev. Mol. Cell Biol. 4: 225-237.

Noda, S., Tanabe, S., and Suzuki, T. (2012). Differential effects of flavonoids on barrier intergrity in human intesinal Caco-2 cells. J. Agric. Food Chem. 60: 4628-4633.

Oshima, T., Miwa, H., and Joh, T. (2008). Aspirin induces gastric epithelial barrier dysfunction by activating p38 MAPK via claudin-7. Am. J. Physiol. Cell Physiol. 295: C800-C806.

Peska, J., and Smolinski, A. (2005). Deoxynivalenol: toxicity and potential effects on humans. J. Toxicol. Environ. Health B Crit. Rev. 8: 39-69.

Pinto Jr, F.E.L., Brandt, C.T., Medeiros, A.C., Oliveira, A.J.F., Jeronimo, S.M., and Brito, H.M.F. (2007). Bacterial translocation in rats nonfunctioning diverted distal colon. Acta Cir. Bras. 22: 195-201.

Pinton, P., Braicu, C., Nougayrede, J.-P., Laffitte, J., Taranu, I., and Oswald, I.P. (2010). Deoxynivalenol impairs porcine intestinal barrier function and decreases the protein expression of claudin- 4 through a mitogen-activated protein kinase-dependent mechanism. J. Nutr. 140: 1956-1962.

Pinton, P., Nougayrède, J.-P., Del Rio, J.-C., Moreno, C., Marin, D.E., Ferrier, L., Bracarense, A.-P., Kolf-Clauw, M., and Oswald, I.P. (2009). The food contaminant deoxynivalenol, decreases intestinal barrier permeability and reduces claudin expression. Toxicol. Appl. Pharm. 237: 41-48.

Pinton, P., and Oswald, I.P. (2014). Effect of deoxynivalenol and other type $B$ trichothecenes on the intestine: a review. Toxins 6: 1615-1643.

Pinton, P., Tsybulskyy, D., Lucioli, J., Laffitte, J., Callu, P., Lyazhri, F., Grosjean, F., Bracarense, A.-P., Kolf-clauw, M., and Oswald, I.P. (2012). Tox- icity of deoxynivalenol and its acetylated derivatives on the intestine: differential effects on morphology, barrier function, tight junctions proteins and MAPKinases. Toxicol. Sci. 130: 180-190.

Pirola, L., and Frojdo, S. (2008). Resveratrol: one molecule, many targets. IUBMB Life 60: 323-332.

Rogoll, D., Bergmann, H., Hellenschmidt, D., Heinze, J., Scheppach, W., Melcher, R., and Richling, E. (2010). Influence of apple polyphenols on the intestinal barrier in a colonic cell model. J. Appl. Bot. Food Qual. 83: 110-117.

Sanchez-Fidalgo, S., Cardeno, A., Villegas, I., Talera, E., and de la Lastra, C.A. (2010). Dietary supplementaton of resveratrol attenuates chronic colonic inflammation in mice. Eur. J. Pharmacol. 633: 78-84.

Scalbert, A., Manach, C., Morand, C., Remesy, C., and Jimenez, I. (2005). Dietary polyphenols and the prevention of diseases. Crit. Rev. Food Sci. Nutr. 45: 287-306.

Schaeffer, H.J., and Weber, M.J. (1999). Mitogen-activated protein kinases: specific messages from ubiquitous messengers. Mol. Cell Biol. 19: 2435-2444.

Schierack, P., Nordhoff, M., Pollmann, M., Weyrauch, K.D., Amasheh, S., Lodemann, U., Jores, J., Tachu, B., Kleta, S., Blikslager, A., Tedin, K., and Wieler, L.H. (2006). Characterization of a porcine intestinal epithelial cell line for in vitro studies of microbial pathogenesis in swine. Histochem. Cell Biol. 125: 293-305.

Schneider, C.A., Rasband, W.S., and Eliceiri, K.W. (2012). NIH Image to ImageJ: 25 years of image analysis. Nat. Methods 9: 671-675.

Sedman, P.C., MacFie, J., and Sagar, P. (1994). The prevalence of gut translocation in humans. Gastroenterology 107: 643-649.

Sergent, T., Parys, M., Garsou, S., Pussemier, L., Schneider, Y.J., and Larondelle, Y. (2006). Deoxynivalenol transport across human intestinal Caco- 2 cells and its effects on cellular metabolism at realistic intestinal concentrations. Toxicol. Lett. 164: 167-176.

Shin, N., Ryu, S., Choi, E., Kang, S., Chang, I., Min, K., and Kim, Y. (1998). Oxyresveratrol as the potent inhibitor on dopa oxidase activity of mushroom tyrosinase. Biochem. Biophys. Res. Commun. 243: 801-803.

Walle, T., Hsieh, F., DeLegge, M.H., Oatis, J.E., and Walle, U.K. (2004). High absorption but very low bioavailability of oral resveratrol in humans. Drug Metabol. Dispos. 32: 1377-1382.

Wang, M., Jin, Y., and Ho, C-T. (1999). Evaluation of resveratrol derivatives as potential antioxidants and identification of a reaction product of resveratrol and 2,2-diphenyl-1-picryhydrazyl radical. J. Agric. Food Chem. 47: 3974-3977.

Xu, L., Liu, C., Xiang, W., Chen, H., Qin, X., and Huang, X. (2014). Advances in the study of oxyresveratrol. Int. J. Pharmacol. 10: 44-54.

Zain, M.E. (2011). Impact of mycotoxins on humans and animals. J. Saudi Chem. Soc. 15: 129-144. 